\title{
Decoherence between the initial and final state radiation in a dense QCD medium
}

\author{
Mauricio Martinez \\ Department of Physics, The Ohio State University, Columbus, OH 43210, USA
}

\begin{abstract}
We study medium modifications to the interference pattern between initial and final state radiation. We compute single gluon production off a highly energetic parton that undergoes a hard scattering and subsequently crosses a dense QCD medium of finite size. Multiple soft scatterings with the medium are resumed within the harmonic oscillator approximation. We find the decoherence of correlated partons traversing the medium depends on two scales: the medium length $L$ and the decorrelation time of the gluon due to the medium $\tau_{f}$. This interplay gives origin to two different asymptotic limits of the gluon spectrum: the coherent $\left(\tau_{f} \gg L\right)$ and incoherent regime $\left(\tau_{f} \ll L\right)$. We discuss the main characteristics of each regime. We show that in both cases there is a gradual onset of decoherence between the initial and final state radiation due to multiple scatterings, that opens the phase space for large angle emissions. We comment on the phenomenological consequences (such as radiative energy loss) of this setup in pA collisions.
\end{abstract}

Keywords: Heavy ion collisions, perturbative QCD, jet quenching

\section{Introduction}

An important ingredient in a jet vacuum parton shower is the role of colour coherence. The basic question addressed by colour coherence is to what extent different emitters in the cascade act independently and the simplest setup to address this question is the quark antiquark antenna. Let us consider for simplicity the colour singlet case and an opening angle $\theta_{\bar{q} q}$ between the quark antiquark pair. The radiation for soft gluons at emission angles $\theta>\theta_{\bar{q} q}$ is suppressed while gluon radiation is basically emitted at $\theta<\theta_{\bar{q} q}$. This simple example is the basis of the angular ordering prescription in jet evolution [1]. In the case when we do not have a non-singlet colour configuration, radiation outside the cone, $\theta>\theta_{\bar{q} q}$, happens with the strength given by the total colour charge i.e. the pair acts as a single emitter with the charge of the pair. This provides a simple probabilistic picture which is implemented in Monte Carlo generators.

The study of the fate of colour coherence when the branching process occurs inside a coloured medium has started recently commonly using an in-medium colour antenna as laboratory [2, 3, 4, 5, 6, 7, 8]. The main findings of these studies can be understood in terms of a modification of the interference coherence pattern of the two emitters in the antenna due to colour rotation in the medium. When the transverse size of the antenna is smaller than the typical colour correlation length inside the medium, the internal colour structure of the antenna and the vacuum colour coherence remains unchanged, i.e., vacuum radiation is angularly ordered. In the opposite limit, when the size of the antenna is similar or larger than the colour correlation length, the medium can effectively decorrelate the pair by colour rotation so that each parton emits medium-induced radiation independently and the angular ordering of the vacuum radiation is broken, i.e., we have antiangular ordering. The antenna radiation in the medium is the basic building block of the recently proposed probabilistic picture of a parton shower in a dense QCD medium $[9,10,11,12]$. 


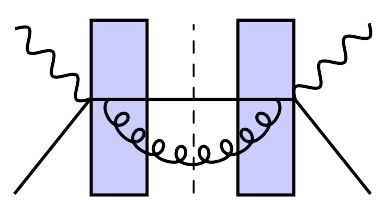

(a)

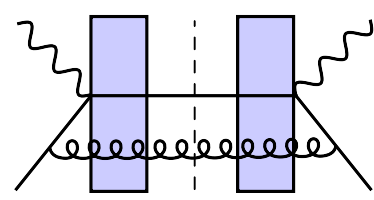

(d)

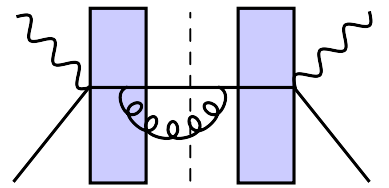

(b)

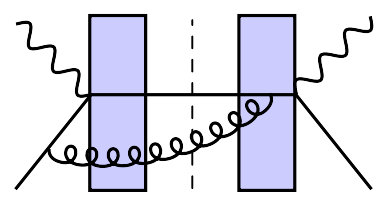

(e)

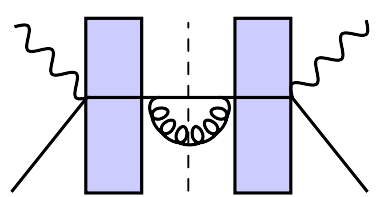

(c)

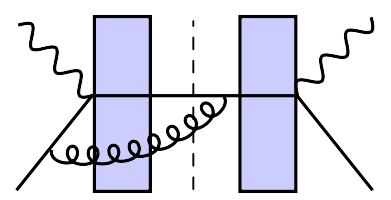

(f)

Figure 1. Different components of the gluon spectrum: (a) the "in-in" component, (b) the "in-out" component, (c) the "out-out" component, (d) the "bef-bef" component, (e) the "bef-in" component, and (f) the "bef-out" component.

In these proceedings contribution we review the extension of medium modifications to the color coherence to another configuration different from final state emissions from an antenna. We focus on the interference between initial and final state radiation.

\section{The gluon spectrum of the initial and final state ratiation}

We study single gluon production off a highly energetic parton that undergoes a hard scattering and subsequently crosses a QCD medium of finite size [13, 14]. This setup was previously studied by us [13] for the case of a finite size dilute medium, in which the interaction with it is modelled through a single hard scattering. Here we shall extend our previous studies obtained at first order in opacity by providing general results for multiple soft scatterings and their specific formulation within the harmonic oscillator approximation. Complete details of the calculation can be found in Ref. [14].

In Fig.1 we show the Feynman diagrams which contribute to the gluon spectrum. Below we briefly describe the main properties of each contribution.

\subsection{Direct emissions}

(i) "Bef-bef": this term can be written as

$$
k^{+} \frac{d N_{b e f-b e f}}{d^{3} k}=\frac{\alpha_{s} C_{F}}{\pi^{2}} \operatorname{Re}\left\{\int \frac{d^{2} \boldsymbol{k}^{\prime}}{(2 \pi)^{2}} \frac{\mathcal{P}\left(\boldsymbol{k}^{\prime}-\boldsymbol{\kappa}, L^{+}\right)}{\boldsymbol{k}^{\prime 2}}\right\},
$$

where $\boldsymbol{\kappa}$ is the transverse momentum of the gluon relative. The function $\mathcal{P}(\boldsymbol{k}, \xi)$ is the probability of having accumulated a certain transverse momentum $\boldsymbol{k}^{2}$ while traversing a longitudinal distance $\xi$ given by

$$
\mathcal{P}(\boldsymbol{k}, \xi)=\frac{4 \pi}{\hat{q} \xi} \exp \left[-\frac{\boldsymbol{k}^{2}}{\hat{q} \xi}\right]
$$

The "bef-bef" contribution (1) is understood as a two-step process: a gluon with momentum $\boldsymbol{k}^{\prime}$ is emitted in the remote past by the incoming parton, the term $1 / \boldsymbol{k}^{2}$ in Eq. (1), and afterwards the gluon follows a random walk while crossing the medium described by $\mathcal{P}\left(\boldsymbol{k}^{\prime}-\boldsymbol{\kappa}, L^{+}\right)$. Gluons from the initial state get their momenta reshuffled and in average, they acquire the maximal amount of accumulated transverse momentum $Q_{s}^{2}=\hat{q} L^{+}$ where $\hat{q}$ is a medium property related with the measure of the transverse momenta per unit lenght. In addition, the multiplicity of gluons associated does not change its value, instead its angular distribution gets smeared out to large angles [14]. 
(ii) "In-in": this contribution corresponds to the emission completely inside the medium by the outcoming parton. The analytical expression is calculated and give us

$$
k^{+} \frac{d N_{i n-i n}}{d^{3} k}=\frac{\alpha_{s} C_{F}}{2 \pi^{2}} \frac{1}{\left(k^{+}\right)^{2}} \operatorname{Re}\left\{2 i k^{+} \int_{0}^{L^{+}} d y^{+} \int \frac{d^{2} \boldsymbol{k}^{\prime}}{(2 \pi)^{2}} \mathcal{P}\left(\boldsymbol{k}^{\prime}-\overline{\boldsymbol{\kappa}}, L^{+}-y^{+}\right) \exp \left((1-i) \frac{\boldsymbol{k}^{\prime 2}}{2 k_{f}^{2}} \tan \left(\Omega y^{+}\right)\right)\right\},
$$

where $k_{f}^{2}=\sqrt{k^{+} \hat{q}}$. Eq. (3) shows that the medium-induced component is also understood as a two-step process. First, the quantum emission of a gluon with momentum $\boldsymbol{k}^{\prime}$ at certain time $\tau_{f} \sim|\Omega|^{-1}$ (the exponential term in the second line of Eq. (3)) and afterwards, the subsequent brownian motion of the gluon along the remaining path through the medium which is described by $\mathcal{P}\left(\boldsymbol{k}^{\prime}-\overline{\boldsymbol{\kappa}}, L^{+}-y^{+}\right)$. The emission spectrum peaks around $k_{f}$ which corresponds to the amount of momentum accumulated during its formation time $\tau_{f}$. The medium-induced component (3) scales with the length of the medium $L^{+}[14]$.

(iii) “In-out": this term takes into account the medium-vacuum interference of the final state radiation and it reads as

$$
k^{+} \frac{d N_{\text {in-out }}}{d^{3} k}=-2 \frac{\alpha_{s} C_{F}}{\pi^{2}} \frac{1}{\overline{\boldsymbol{\kappa}}^{2}} \operatorname{Re}\left\{1-\exp \left[-i \frac{\overline{\boldsymbol{\kappa}}^{2}}{2 k^{+} \Omega} \tan \left(\Omega L^{+}\right)\right]\right\}
$$

This contribution becomes important when emissions take place near to the boundary of the medium [14].

(iv) "Out-out": this contribution takes place completely outside the medium due to bremmstrahlung of the outcoming parton and is given by [14]

$$
k^{+} \frac{d N_{\text {out }- \text { out }}}{d^{3} k}=\frac{\alpha_{s} C_{F}}{\pi^{2}} \frac{1}{\overline{\boldsymbol{\kappa}}^{2}}
$$

\subsection{Interferences}

The information about the colour correlation between both emitters is carried out by the interference terms " $b e f$ in" and "bef-out". The color correlation between both emitters will depend on whether the QCD medium is able to resolve the quark-gluon system or not. This is analogous to the studied case of the $q \bar{q}$ antenna immersed in a QCD medium $[2,7,4,6,3]$, with $r_{\perp}$ the transverse $q \bar{q}$ dipole size. However, the transverse size of the quark-gluon system depends on the accumulated transverse momentum of the gluon at a certain longitudinal position inside the medium due to its brownian motion [14].

(i) "Bef-in": this term can be expressed as follows

$$
\begin{aligned}
k^{+} \frac{d N_{b e f-i n}}{d^{3} k} & =-2 \frac{\alpha_{s} C_{F}}{\pi^{2}} \operatorname{Re}\left(i \int_{0}^{L^{+}} d y^{+} \int \frac{d^{2} \boldsymbol{k}^{\prime}}{(2 \pi)^{2}} \frac{\boldsymbol{k}^{\prime} \cdot\left(\boldsymbol{k}^{\prime}-\delta \boldsymbol{k} \cos \left(\Omega y^{+}\right)\right)}{\left(\boldsymbol{k}^{\prime}-\delta \boldsymbol{k} \cos \left(\Omega y^{+}\right)\right)^{2}}\right. \\
& \times \frac{\mathcal{P}\left(\boldsymbol{k}^{\prime}-\overline{\boldsymbol{\kappa}}, L^{+}-y^{+}\right)}{2 k^{+}} \exp \left\{(1-i) \frac{\boldsymbol{k}^{\prime 2}}{k_{f}^{2}} \tan \left(\Omega y^{+}\right)\right\}\left[1-\exp \left\{i \frac{\left(\boldsymbol{k}^{\prime}-\delta \boldsymbol{k} \cos \left(\Omega y^{+}\right)\right)^{2}}{2 k^{+} \Omega \sin \left(\Omega y^{+}\right) \cos \left(\Omega y^{+}\right)}\right\}\right) .
\end{aligned}
$$

(ii) 'Bef-out": this term can be written as follows

$$
\begin{aligned}
k^{+} \frac{d N_{\text {bef-out }}}{d^{3} k} & =-2 \frac{\alpha_{s} C_{F}}{\pi^{2}} \operatorname{Re}\left\{\frac{\overline{\boldsymbol{\kappa}} \cdot\left(\overline{\boldsymbol{\kappa}}-\delta \boldsymbol{k} \cos \left(\Omega L^{+}\right)\right)}{\overline{\boldsymbol{\kappa}}^{2}\left(\overline{\boldsymbol{\kappa}}-\delta \boldsymbol{k} \cos \left(\Omega L^{+}\right)\right)^{2}} \exp \left[(1-i) \frac{\overline{\boldsymbol{\kappa}}^{2}}{2 k_{f}^{2}} \tan \left(\Omega L^{+}\right)\right]\right. \\
& \left.\times\left(1-\exp \left[i \frac{\left(\overline{\boldsymbol{\kappa}}-\delta \boldsymbol{k} \cos \left(\Omega L^{+}\right)\right)^{2}}{2 k^{+} \Omega \sin \left(\Omega L^{+}\right) \cos \left(\Omega L^{+}\right)}\right]\right)\right\} .
\end{aligned}
$$

The asymptotic behaviour of both terms (6) and (7) is determined by how large or small is the argument of the phases. As a consequence two different limits arise: the coherent and the incoherent regime [14]. Despite their differences, both regimes share similar features as we shall discuss below. 


\subsection{Coherent regime: $L^{+} \ll \tau_{f}$}

This limit corresponds to the physical situation when the emitted gluon remains coherent during all the time while crossing the QCD medium [14]. The medium acts as a unique scattering center and thus the effective momentum transfer is $Q_{s}^{2}=\hat{q} L^{+}$. The gluon spectrum is reduced to the "bef-bef", "bef-out" and "out-out" terms (see Fig.(1)). Interferences are completely suppressed when $|\delta \boldsymbol{k}| \ll Q_{s}$ so there are gluon emissions outside of the angle associated to the hard scattering $\theta_{q q}$ and hence, antiangular ordering. The typical momentum scale transferred by the medium $Q_{s}^{2}=\hat{q} L^{+}$sets up a upper bound for large angle radiation, so angular emissions lie in the range $\theta_{q q} \leq \theta \leq \theta_{\max }$ where $\theta_{\max } \sim Q_{s} / k^{+}$. The spectrum is completely suppressed above $\theta_{\max }$ and the vacuum coherence pattern is recovered, i.e., when $|\delta \boldsymbol{k}| \gg Q_{s}$ gluons are radiated in a coherent manner as in the vacuum [14].

\subsection{Incoherent regime: $L^{+} \gg \tau_{f}$}

In this limit, all the components of the spectrum (see Fig.(1)) must be considered. In a similar manner to the coherent regime, there is a partial suppression of interferences between both emitters due to the multiple scatterings in the medium. Large angle gluon emissions are expected in the kinematic region $\theta_{q q} \leq \theta \leq \theta_{\max }$ and the vacuum coherence pattern is again reestablished for $|\boldsymbol{k}|>Q_{s}$.

\section{Conclusions}

In this contribution we review our recent work on the modifications to the interference pattern between the initial and final state radiation due to the presence of a QCD medium [14]. We obtain an analytical expresion for the medium induced gluon spectrum which is composed by the independent gluon emissions associated with the incoming and outgoing parton as well as the interference terms. The color connection between the initial and final state depends on the time-dependent transverse distance of the quark-gluon system. We discuss the incoherent and coherent regimes of the gluon spectrum. In both limits we observe a partial loss of coherence due to the medium interactions independent of the medium. Therefore, medium interactions opens the phase space for large angle emissions, e.g. antiangular ordering, up to a maximal angle $\theta_{\max }=Q_{s} / k^{+}$determined by the medium properties. The vacuum coherence is recovered for angle emissions above $\theta>\theta_{\max }$. Our results may have relevant phenomenological consequences on hadron production in nuclear collisions specially in the dilute-dense regime, e.g., pA collisions.

Acknowledgments The author thanks to T. Altinoluk, N. Armesto, G. Beuf, H. Ma, Y. Mehtar-Tani and C. Salgado for valuable discussions. This work was supported by U.S. Department of Energy under Grant No. DE-SC0004286.

\section{References}

[1] Y. L. Dokshitzer, V. A. Khoze, A. H. Mueller, S. I. Troian, Basics of Perturbative QCD, Gif-sur-Yvette, France: Ed. Frontieres (1991) 274 p.

[2] Y. Mehtar-Tani, C. A. Salgado, K. Tywoniuk, Antiangular Ordering of Gluon Radiation in QCD Media, Phys. Rev. Lett. 106 (2011) 122002.

[3] Y. Mehtar-Tani, C. A. Salgado, K. Tywoniuk, Jets in QCD Media: from Color Coherence to Decoherence, Phys. Lett. B707 (2012) 156-159.

[4] Y. Mehtar-Tani, C. A. Salgado, K. Tywoniuk, The radiation pattern of a QCD antenna in a dilute medium, JHEP 1204 (2012) 064.

[5] Y. Mehtar-Tani, K. Tywoniuk, Jet coherence in QCD media: the antenna radiation spectrum, JHEP 1301 (2013) 031.

[6] Y. Mehtar-Tani, C. A. Salgado, K. Tywoniuk, The Radiation pattern of a QCD antenna in a dense medium, JHEP 1210 (2012) 197.

[7] J. Casalderrey-Solana, E. Iancu, Interference effects in medium-induced gluon radiation, JHEP 1108 (2011) 015.

[8] J. Casalderrey-Solana, Y. Mehtar-Tani, C. A. Salgado, K. Tywoniuk, New picture of jet quenching dictated by color coherence, Phys.Lett. B725 (2013) 357-360.

[9] J.-P. Blaizot, F. Dominguez, E. Iancu, Y. Mehtar-Tani, Medium-induced gluon branching, JHEP 1301 (2013) 143, doi: 10.1007/JHEP01(2013)143.

[10] J.-P. Blaizot, E. Iancu, Y. Mehtar-Tani, Medium-induced QCD cascade: democratic branching and wave turbulence, Phys.Rev.Lett. 111 (2013) 052001, doi:10.1103/PhysRevLett.111.052001.

[11] J.-P. Blaizot, F. Dominguez, E. Iancu, Y. Mehtar-Tani, Probabilistic picture for medium-induced jet evolution, JHEP 1406 (2014) 075, doi: 10.1007/JHEP06(2014)075.

[12] L. Apolinario, N. Armesto, J. G. Milhano, C. A. Salgado, Medium-induced gluon radiation and colour decoherence beyond the soft approximation, arXiv:1407.0599

[13] N. Armesto, H. Ma, M. Martinez, Y. Mehtar-Tani, C. A. Salgado, Interference between initial and final state radiation in a QCD medium, Phys.Lett. B717 (2012) 280-286.

[14] N. Armesto, H. Ma, M. Martinez, Y. Mehtar-Tani, C. A. Salgado, Coherence phenomena between initial and final state radiation in a dense QCD medium, JHEP 1312 (2013) 052. 